Aus der geburtshülflich-gynäkologischen Klinik des Professor Dr. Rudolf Chrobak in Wien.

II.

\title{
Ueber das Verhältniss gewisser Durchmesser des kindlichen und des mütterlichen Schädels.
}

Von

\author{
Dr. Bronisław v. Skałkowski.
}

Als Fortsetzung meiner in diesem Archive, Bd. XXXVIII, Hft. 3 erschienenen Arbeit über das Verhältniss gewisser Durchmesser des kindlichen und des mütterlichen Schädels, welche $\mathrm{Zu}$ sammenstellung sich auf reife Kinder bezog, erlaube ich mir in Nachstehendem das Ergebniss meiner Untersuchungen über das Verbalten der Durchmesser der Schädel frühgeborener Kinder zu. jenen der Mütter in Kürze mitzutheilen.

Es wurden bei dieser Arbeit 100 Mutterköpfe und deren Kinder gemessen. Da aber $11 \mathrm{Mal}$ Zwillinge und 1 Mal Drillinge vorkamen, so wurden zusammen 213 Köpfe gemessen, nämlich : 100 mütterliche und 113 kindliche Schädel. Nach der Zahl der vorausgegangenen Geburten gruppirt, kamen zur Untersuchung: 59 Ip., 24 IIp., 9 III p., 2 IV p., 3 Vp., 1 VIp., 1 VIIp. und 1 VIIIp., zusammen 100 Mütter. Mit Rücksicht auf den gemischten Menschenschlag, aus dem sich das Material des Wiener Gebärhauses zusammensetzt, und in weiterer Rücksicht darauf, dass sich nach meiner früheren Arbeit keine verwerthbaren Ergebnisse bezüglich der Nationalität ergeben haben, wurde diesmal bei der Zusammenstellung der Tabellen die Nationalität nicht mehr berücksichtigt.

Diesmal handelte es sich nur um das Verhältniss der Schädel der frühgeborenen Kinder zu jenen der Mütter. In der früheren Zusammenstellung berücksichtigte ich nur reife Kinder, und als reif 
betrachtete ich solche, welche bei sonst regelmässiger Körpergestaltung ein Gewicht von wenigstens $3000 \mathrm{~g}$ hatten. Genügend sichere Merkmale für die Reife oder Unreife des Kindes giebt es nicht. Die Berechuung der Dauer der Schwangerschaft nach dem Tage der Empfängniss ist für eine grössere Zusammenstellung bei den Schwangeren an unserer Gebäranstalt nicht durchführbar. Die Dauer der Schwangerschaft bach dem Auftreten der letzten Menstruation zu bestimmen, ist auch schwer, schon deshalb, weil es wenig Mütter giebt, welche den Zeitpunkt des Eintrittes der letzten Menstruation mit voller Sicherheit angeben können. Ich musste mich deshalb auf den rein objectiven Befund beschränken und als Maassstab zur Beurtheilung, ob ein Kind frühgeboren sei oder nicht, jenes Gewicht desselben annehmen, welches das Kind am ersten Tage zwei bis drei Stunden nach der Geburt hatte.

Verschiedene Forscher nun geben verschiedene Zahlen als Durchschnittsgewichte für reife Kinder an. So z. B. findet G. Veit für Knaben $3545 \mathrm{~g}$, für Mädchen $3440 \mathrm{~g}$; Hecker für Knaben 3310, für Mädchen $3230 \mathrm{~g}$; Scanzoni für Knaben 3530, für Mädchen $3430 \mathrm{~g}$; Schroeder bei einer Länge von $49 \mathrm{~cm} \mathrm{im}$ Durchschnitte 3179 und Spiegel berg $3128 \mathrm{~g}$.

Das sind Zahlen, welche nicht den Durehschnittszahlen der im Wiener Gebärhause zur Welt gebrachten Kinder entsprechen. Da mir von vornherein die oben angegebenen Zahlen zu gross schienen, ich aber andererseits aus den früher angegebenen Gründen keine verlässlichen Forschungen anstellen konnte, so berechnete ich das Durchschnittsgewicht von 1000 Kindern der Klinik (500 Knaben und 500 Mädchen) ohne Rücksicht auf ihre Reife und fand für die ersten 3111 g, für die anderen $2998 \mathrm{~g}$, als Durchschnittsgewicht eines an der Klinik geborenen Kindes $3054,50 \mathrm{~g}$.

Wenn ich zu meinen Messungen nur solche Kinder auswählte, welche das höchste Gewicht von $2700 \mathrm{~g}$ hatten, so glaube ich nicht zu hoch gegriffen zu haben, um für die Verhältnisse des Wiener Gebärhauses solche Kinder mit Recht als frühgeboren bezeichnen zu dürfen. Das würde auch mit den Zahlen von Ahlfeld übereinstimmen, welcher für die 40 . Woche $3168 \mathrm{~g}$ angiebt und Kinder mit $2753 \mathrm{~g}$ in die 35. und mit $2424 \mathrm{~g}$ in die 34 . Woche verlegt; demnach würden die Kinder, welche ich gemessen habe, also solche von einem Gewichte unter $2700 \mathrm{~g}$, als wenigstens noch 1 Monat vom Ende ihrer Entwicklung entfernt, mit Recht als frühgeborene Kinder zu bezeichnen sein. 
Um Wiederholungen zu vermeiden, berufe ich mich auf meine Arbeit über Messungen an reifen Kindern. Auch wurden die jetzigen Messungen genau ebenso vorgenommen, wie damals. Das kleinste gemessene Kind wog 1400, die Mehrzahl entfällt auf $2000-2500 \mathrm{~g}$ Gewicht. Die überwiegende Mehrzahl der gemessenen Kinder wurde von selbst in Schädellage geboren. Nur einige von den Zwillingen, sowie die Drillingskinder kamen in Beckenendlage zur Welt. Diesen Umstand halte ich für unerheblich, weil ich die Messungen erst am zweiten Tage nach der Geburt gemacht habe, also zu einer Zeit, in der die Veränderungen, welche der Schädel während der Geburt erlitten hatte, sicher verschwunden waren, und da ferner zu berücksichtigen ist, dass solche Veränderungen überhaupt an den Schädeln frühgeborener Kinder wegen der Leichtigkeit der Geburt und der Kürze ihrer Dauer verhältnissmässig unbedeutend sind.

Ich maass die Diameter bitemporalis, biparietalis und suboccipito-bregmatica. Im Grossen und Ganzen war der Gang meiner Untersuchungen, was die Bearbeitung der Messzahlen betrifft, derselbe, wie 'bei der Arbeit über die Schädel der reifen Kinder, nur fügte ich noch eine Tabelle, welche auch das Gewicht der Kinder und dessen Verhältnisse zum Kopfdurchmesser beleuchtet, hinzu.

Die ersten drei Tabellen zeigen, wie oft bei einer bestimmten Grösse des Durchmessers am mütterlichen Schädel ein Durchmesser von bestimmter Grösse des kindlichen Schädels vorgekommen ist (s. S. 248-250).

Vergleicht man diese Tabellen mit jenen, welche über die Köpfe reifer Kinder zusammengestellt wurden, so ist für den ersten Augenblick ein auffallender Unterschied vorhanden. Die Regelmässigkeit, mit der bei einer gewissen Grösse des Durchmessers am mütterlichen Schädel ein Durchmesser des kindlichen Schädels von einer bestimmten Grösse erschienen ist, ist fast ganz verschwunden. Diese Thatsache würde ihre Erklärung darin finden, dass, nachdem es sich um die Schädel von Frühgeburten handelt, deren Grösse bezw. Gewicht in Grenzen von 1400 bis $2700 \mathrm{~g}$ schwankte, Schädel vorgekommen sind, die verhältnissmässig noch klein und untereinander, was die Grösse betrifft, vielfach verschieden waren. Die Regelmässigkeit also, die man bei den Schädeln reifer Kinder vorfand, mit welcher bei einer gewissen Grösse des mütterlichen Schädels kindliche Köpfe mit entsprechend grossen Durchmessern vorkamen, konnte man hier von vornherein 
Tabelle I. Verhältniss der Diameter bitemporalis der Mutter zu jener des Kindes.

\begin{tabular}{|c|c|c|}
\hline $\begin{array}{l}\text { Bei einer Grösse des } \\
\text { mütterlichen Kopfdurch- } \\
\text { messers von cm }\end{array}$ & $\begin{array}{l}\text { ist vorgekommen der } \\
\text { Durchmesser des kind- } \\
\text { lichen Schädels in der } \\
\text { Grösse von cm }\end{array}$ & wieviel Mal? \\
\hline 11,5 & 7,5 & 5 \\
\hline 12 & $\begin{array}{l}6,5 \\
7 \\
7,5 \\
8\end{array}$ & $\begin{array}{r}6 \\
12 \\
5 \\
1\end{array}$ \\
\hline 12,5 & $\begin{array}{l}6,5 \\
7 \\
7,5 \\
8 \\
8,5\end{array}$ & $\begin{array}{l}3 \\
7 \\
9 \\
7 \\
1\end{array}$ \\
\hline 13 & $\begin{array}{l}6,5 \\
7 \\
7,5 \\
8\end{array}$ & $\begin{array}{r}1 \\
7 \\
12 \\
8\end{array}$ \\
\hline 13,5 & $\begin{array}{l}7 \\
7,5 \\
8 \\
8,5\end{array}$ & $\begin{array}{l}4 \\
7 \\
2 \\
3\end{array}$ \\
\hline 14 & $\begin{array}{l}7,5 \\
8\end{array}$ & $\begin{array}{l}5 \\
4\end{array}$ \\
\hline 14,5 & $\begin{array}{l}7 \\
7,5 \\
8\end{array}$ & $\begin{array}{l}1 \\
1 \\
1\end{array}$ \\
\hline 15 & 7,5 & 1 \\
\hline & $\overline{\text { Summa }}$ & 113 \\
\hline
\end{tabular}

nicht erwarten, da es sich fast immer um noch nicht ausgebildete Köpfe handelte. Es ist jedoch trotzdem das verhältnissmässig gleichbleibende Verhalten der Diameter biparietalis auffallend, wie aus der Tabelle II zu ersehen ist. Unter 113 Fällen, bei denen die Grösse des mütterlichen Durchmessers $14-15 \mathrm{~cm}$ betrug, ist am kindlichen Schädel derselbe Durchmesser in der Grösse von $9 \mathrm{~cm} 59 \mathrm{Mal}$ vorgekommen. Das, glaube ich, weist mit grosser Sicherheit darauf hin, dass eben die Diameter biparietalis jener Durchmesser am kindlichen Schädel ist, welcher am frühesten seine annähernde Grösse erreicht und bezüglich seiner Grösse schon an Schädeln frühgeborener Kinder die grösste Beständigkeit zeigt. Dieser Umstand veranlasste mich zu prüfen, wie oft sich 
Tabelle 11. Verhältniss der Diameter biparietalis der Mutter zu jener des Kindes.

\begin{tabular}{|c|c|c|}
\hline $\begin{array}{l}\text { Bei einer Grösse des } \\
\text { mütterlichen Kopfdurch- } \\
\text { messers von cm }\end{array}$ & $\begin{array}{l}\text { ist vorgekommen der } \\
\text { Durchmesser des kind- } \\
\text { lichen Schädels in der } \\
\text { Grösse von cm }\end{array}$ & wieviel Mal? \\
\hline 13 & 8 & 2 \\
\hline 14 & $\begin{array}{c}7,5 \\
8 \\
8,5 \\
9 \\
9,5 \\
10\end{array}$ & $\begin{array}{r}1 \\
1 \\
4 \\
13 \\
1 \\
1\end{array}$ \\
\hline 14,5 & $\begin{array}{c}7,5 \\
8 \\
8,5 \\
9 \\
9,5 \\
10\end{array}$ & $\begin{array}{r}1 \\
3 \\
7 \\
14 \\
2 \\
1\end{array}$ \\
\hline 15 & $\begin{array}{l}7 \\
8 \\
8,5 \\
9 \\
9,5\end{array}$ & $\begin{array}{r}1 \\
2 \\
9 \\
32 \\
7\end{array}$ \\
\hline 15,5 & $\begin{array}{l}8,5 \\
9\end{array}$ & $\begin{array}{l}1 \\
4\end{array}$ \\
\hline 16 & $\begin{array}{l}8,5 \\
9 \\
9,5\end{array}$ & $\begin{array}{l}2 \\
2 \\
1\end{array}$ \\
\hline 16,5 & 8 & 1 \\
\hline & Summa & 113 \\
\hline
\end{tabular}

die Diameter biparietalis in einer bestimmten Grösse bei entsprechendem Gewichte der Kinder zeigt. Ich legte also eine grosse Tabelle an, welche ich dann übersichtshalber zusammenzog, und zwar auf drei Abtheilungen, in welche die Kinder ihren Gewichten nach eingereiht wurden, und zwar I. Gruppe von 1400, als dem kleinsten Kinde, das ich gemessen habe, bis $2000 \mathrm{~g}$, dann die zweite Gruppe von 2000-2350 g und schliesslich die dritte von $2350-2700 \mathrm{~g}$, als dem grössten Gewichte meiner gemessenen Kinder. Das Ergebniss ist in Tab. IV (S. 250) zusammengestellt. 
Tabelle III. Verhältniss der Diameter suboccipitobregmatica der Mutter zu jener des Kindes.

\begin{tabular}{|c|c|c|}
\hline $\begin{array}{l}\text { Bei einer Grösse des } \\
\text { mütterlichen Kopfdurch- } \\
\text { messers von cm }\end{array}$ & $\begin{array}{l}\text { ist vorgekommen der } \\
\text { Durchmesser des kind- } \\
\text { lichen Schädels in der } \\
\text { Grösse von } \mathrm{em}\end{array}$ & wieviel Mal? \\
\hline 14 & 9 & 5 \\
\hline 14,5 & $\begin{array}{l}8,5 \\
9 \\
9,5\end{array}$ & $\begin{array}{l}1 \\
6 \\
1\end{array}$ \\
\hline 15 & $\begin{array}{c}8 \\
8,5 \\
9 \\
9,5 \\
10\end{array}$ & $\begin{array}{r}1 \\
5 \\
25 \\
8 \\
1\end{array}$ \\
\hline 15,5 & $\begin{array}{r}8,5 \\
9 \\
9,5 \\
10 \\
\end{array}$ & $\begin{array}{r}3 \\
11 \\
8 \\
1\end{array}$ \\
\hline 16 & $\begin{array}{c}8 \\
8,5 \\
9 \\
9,5 \\
10\end{array}$ & $\begin{array}{r}1 \\
1 \\
18 \\
4 \\
2\end{array}$ \\
\hline 16,5 & $\begin{array}{l}9 \\
9,5\end{array}$ & $\begin{array}{l}4 \\
3 \\
\end{array}$ \\
\hline 17 & $\begin{array}{l}8,5 \\
9 \\
9,5\end{array}$ & $\begin{array}{l}1 \\
1 \\
2\end{array}$ \\
\hline & Summa & 113 \\
\hline
\end{tabular}

Tabelle IV.

Bei einem Gawichte von

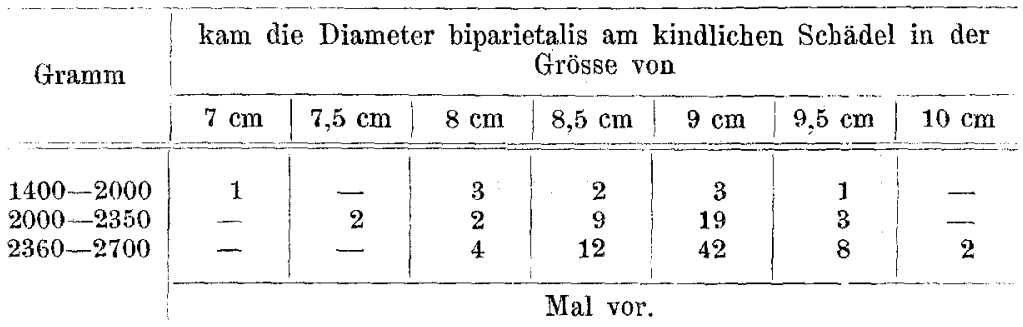

Tabelle IV zeigt, dass man bei einer Grösse des Kindes, dessen Gewicht zwischen 2360-2700 g wechselt, in 62 Fällen auf eineu Durchmesser biparietalis von $8,5-9,5 \mathrm{~cm}$ rechnen darf, und 
andererseits, dass bei diesem Gewichte der Kinder bezw. dieser Grösse die Diameter biparietalis die grösste Gleichmässigkeit in Bezug auf Länge zeigt, dass sie also ein Durchmesser ist, der am schnellsten seine Grösse erreicht. Diese Thatsache hat schon Schroeder beobachtet und auf sie aufmerksam gemacht. Da wir jedoch vor der Geburt ebensowenig über das Gewicht des Kindes, wie über seine Grösse unterrichtet sind, und wir andererseits den gleichen Gang der Untersuchung, wie ich ihn bei den Schädeln reifer Kinder gemacht habe, beizubehalten wünschten, stellte ich die Tabelle $\mathrm{V}$ zusammen, um zu erfragen, wie oft eine Zahl als Unterschied zwischen der Grösse des mütterlichen und des kindlichen Kopfdurchmessers vorgekommen ist, und zwar für alle drei Durchmesser, die Diameter bitemporalis, biparietalis und suboccipito-bregmatica.

Tabelle V. Die Unterschiede zwischen den gleichnamigen Durchmessern am Schädel der Mutter und des Kindes.

\begin{tabular}{r|c|c|c|c}
\hline Nr. & $\begin{array}{c}\text { Unterschied } \\
\text { in cm }\end{array}$ & Bitemporalis & Biparietalis & $\begin{array}{c}\text { Suboccipito- } \\
\text { bregmatica }\end{array}$ \\
\hline & 3,5 & 1 & - & - \\
2 & 4 & 6 & 1 & - \\
3 & $\mathbf{4}$ & $\mathbf{1 1}$ & 1 & 9 \\
4 & 5 & 38 & 28 & 13 \\
5 & 5,5 & 12 & 40 & 36 \\
6 & 6 & $\mathbf{1 1}$ & 15 & 20 \\
7 & 6,5 & $\mathbf{2}$ & 6 & 24 \\
8 & 7 & - & 2 & 8 \\
9 & 7,5 & - & 1 & 1 \\
10 & 8 & $\mathbf{1 1 3}$ & 113 & 113 \\
11 & 8,5 & & &
\end{tabular}

Aus dieser Tabelle ersieht man, dass der Unterschied von 5-6 $6 \mathrm{~cm}$ zwischen der Grösse der mütterlichen Bitemporalis und jener der kindlichen in 113 Fällen 82 Mal vorgekommen ist, $d . h$. in 72,5 Proc.

Ein Unterschied von derselben Grösse bei der Diameter biparietalis kam auf 113 Fälle 85 Mal vor, das ist in 75,2 Proc., und ein Unterschied von $6-7 \mathrm{~cm}$ bei der Diameter suboccipitobregmatica auf 113 Fälle $80 \mathrm{Mal}$ vor, das ist in 70,7 Proc.

Fassen wir die oben angeführten Beobachtungen in Kürze zusammen, so zeigt der Schädel frühgeborener Kinder gegenüber 
252 v. Skalkowski, Jeber d, Verhältniss gewisser Durchmesser u. s. w.

dem mütterlichen Schädel desto grössere Aehnlichkeit, je grösser das Kind ist, und von allen drei Durchmessern ist es hauptsächlich die Diameter biparietalis, welche am frühesten ihre Grösse erreicht, andererseits die Diameter bitemporalis, jene, welche geringere Gleichmässigkeit zeigt und nicht immer gleichen Schritt hält mit dem Wachsthume der Diameter biparietalis. Wollte man aus diesen Messungen einen praktisch verwerthbaren Schluss ziehen, so müsste man sagen, dass wir bei Frühgeburten noch weniger als bei reifen Kindern ein annähernd sicheres Verhältniss der verschiedenen Durchmesser zu treffen erwarten können, dass es aber immerhin einen gewissen Werth hat, zu wissen, dass der geburtshülflich wichtigste Durchmesser des kindlichen Schädels, die Diameter biparietalis, in etwa 75,2 Proc. um $5-6 \mathrm{~cm}$ kleiner vorgefunden wurde, als der Durchmesser am mütterlichen Schädel, und dass dieser Durchmesser bei Kindern von 2300 bis $2700 \mathrm{~g}$ Gewicht in 68 Fällen $62 \mathrm{Mal}$ eine Grösse von $8,5-9,5 \mathrm{~cm}$ gezeigt hat und nur $2 \mathrm{Mal}$ ein Maass von $10 \mathrm{~cm}$ aufwies. 